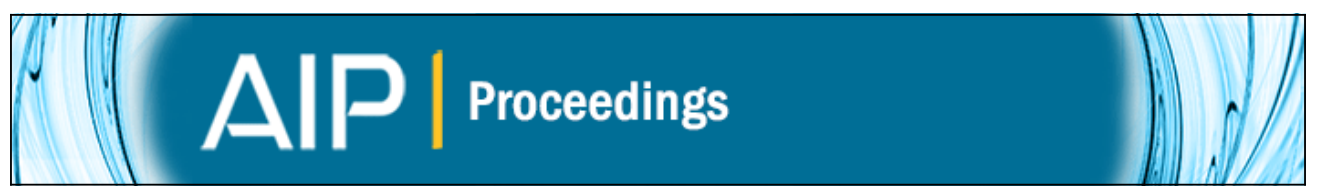

\title{
Frequency Domain Tomography Of Evolving Laser-Plasma Accelerator Structures
}

Peng Dong, Stephen Reed, Serguei Kalmykov, Gennady Shvets, and Mike Downer

Citation: AIP Conference Proceedings 1086, 131 (2009); doi: 10.1063/1.3080893

View online: http://dx.doi.org/10.1063/1.3080893

View Table of Contents:

http://scitation.aip.org/content/aip/proceeding/aipcp/1086?ver=pdfcov

Published by the AIP Publishing

\section{Articles you may be interested in}

Summary Report of Working Group 1: Laser-Plasma Acceleration

AIP Conf. Proc. 1299, 79 (2010); 10.1063/1.3520427

Hybrid Laser-Plasma Wakefield Acceleration

AIP Conf. Proc. 1299, 483 (2010); 10.1063/1.3520370

Laser-Plasma Wakefield Acceleration with Higher Order Laser Modes

AIP Conf. Proc. 1299, 197 (2010); 10.1063/1.3520313

Frequency-Domain Streak Camera and Tomography for Ultrafast Imaging of Evolving and Channeled Plasma Accelerator Structures

AIP Conf. Proc. 1299, 121 (2010); 10.1063/1.3520297

Studies of laser wakefield structures and electron acceleration in underdense plasmasa)

Phys. Plasmas 15, 056703 (2008); 10.1063/1.2856373 


\title{
Frequency Domain Tomography Of Evolving Laser-Plasma Accelerator Structures
}

\author{
Peng Dong ${ }^{a}$, Stephen Reed ${ }^{\mathrm{a}}$, Serguei Kalmykov ${ }^{\mathrm{a}}$, Gennady Shvets ${ }^{\mathrm{a}}$ and \\ Mike Downer ${ }^{\mathrm{a}}$ \\ ${ }^{a}$ Department of Physics, University of Texas at Austin
}

\begin{abstract}
Frequency Domain Holography (FDH), a technique for visualizing quasistatic objects propagating near the speed of light, has produced "snapshots" of laser wakefields, ${ }^{1}$ but they are averaged over structural variations that occur during propagation through the plasma medium. Here we explore via simulations a generalization of FDH --- that we call Frequency Domain Tomography (FDT) --- that can potentially record a time sequence of quasistatic snapshots, like the frames of a movie, of the wake structure as it propagates through the plasma. FDT utilizes a several probe-reference pulse pairs that propagate obliquely to the drive pulse and wakefield, along with tomographic reconstruction algorithms similar to those used in medical CAT scans.
\end{abstract}

Keywords: laser, plasma, wakefield, frequency-domain holography, CAT.

PACS: $52.38 . \mathrm{Kd}$

\section{INTRODUCTION}

We recently reported detailed "snapshots" of laser wakefield accelerating structures ${ }^{1}$ captured by Frequency Domain Holography (FDH), ${ }^{2}$ a single-shot visualization technique in which a probe-reference pulse pair propagates collinearly through the plasma with a relativistically intense drive pulse and its wakefield. The probe pulse is temporally lengthened by chirping so that it illuminates the entire wakefield at once, like the "object" beam of conventional holography. It then interferes at the detection plane of an imaging spectrometer with a similarly chirped "reference" pulse that propagated in front of the drive pulse, thereby recording a frequency-domain interferogram (or hologram) that encodes the phase structure of the wake. The hologram is "read" electronically by a Fourier transform procedure to reconstruct the object.

A limitation of this technique is that longitudinal variations of the accelerating structure are averaged as the drive pulse propagates through the plasma. Specifically, writing the plasma structure as an electron density profile $n_{e}(r, \zeta, z)$, and the corresponding local index of refraction as $\eta(r, \zeta, z)$, the phase change imprinted on the probe pulse is an integral $\Delta \phi(r, \zeta)=\frac{2 \pi}{\lambda_{p r}} \int_{0}^{l}[1-\eta(r, \zeta, z)] d z$. Here $r$ denotes distance from the drive pulse propagation axis, $\zeta$ distance behind the drive pulse, and $z$ the propagation distance from the gas jet entrance, and we have assumed for simplicity that the wake is cylindrically symmetric. Many kinds of longitudinal $(z)$ variations can cause the holographically reconstructed $\Delta \phi(r, \zeta)$ to deviate from the actual plasma 
wakefield shapes $n_{e}(r, \zeta, z)$ : laser focusing and defocusing, gas jet density variation, wave breaking and beam loading. In the bubble regime, ${ }^{3}$ this is especially serious because bubbles can form rapidly following self-focusing and steepening of the drive pulse. ${ }^{4}$ Conventional FDH averages over this temporal sequence, preserving no record of the bubble's temporal evolution.

An additional practical limitation of conventional FDH is that forward-directed white light continuum or optical harmonics generated by the pump can interfere with the probe and reference, which are typically at a different wavelength than the pump to enable separation from pump light. Such undesired background light inhibits image reconstruction, especially in dense plasmas where "bubble" structures are important. For example, in previous experiments, ${ }^{1}$ white light continuum made image reconstruction impossible at plasma densities above $5.9 \times 10^{18} \mathrm{~cm}^{-3}$. The white light is mainly restricted to the cone of the pump pulse.

\section{FREQUENCY DOMAIN STREAK CAMERA}

As a first step toward resolving these limitations, we propose augmenting the collinear probe-reference pulse pair with a non-collinear probe-reference pulse pair that propagates at angle $\alpha$ relative to the pump pulse. For example, Fig. 1 shows an oblique probe interacting with a plasma bubble. For sufficiently large angles $\alpha$ the white light cone is avoided. Moreover, the phase "streak" imprinted on the probe pulse profile (see Fig. 1b,c) chronicles evolution of the bubble, which traverses an oblique lateral path across the probe pulse profile (Fig. 1b). During this passage, any change of the bubble shape or density changes the width and depth of the phase streak on the probe pulse as a function of time and transverse spatial dimension. The phase streak is then recovered (Fig. 1c) from interference between the reference and probe pulse as in conventional FDH. We call this the Frequency Domain Streak Camera (FDSC) because temporal evolution of the bubble is converted onto a phase streak on the probe pulse. This temporal phase information can be used to interpret a longitudinally averaged snapshot obtained by conventional FDH.
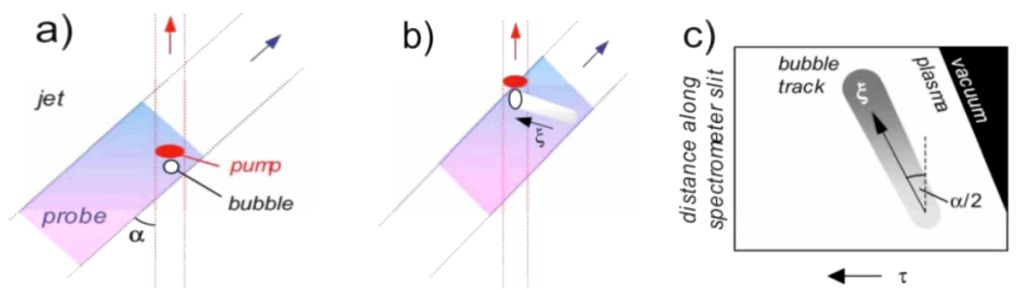

FIGURE 1. (a) Snapshot of pump (red), trailing bubble (white) and chirped probe overlapping at angle $\alpha$ in gas jet; (b) same at later time, showing how the evolving bubble sweeps out a stripe (gray) tilted at angle $\alpha / 2$ from the leading edge of the probe; (c) schematic reconstructed probe phase shift $\Delta \Phi_{\mathrm{pr}}(\rho, \tau)$, where $\rho$ denotes distance perpendicular to the propagation axis, and $\tau$ time behind the leading edge, of the probe pulse. The spectrometer slit must lie along $\rho$ to record this data. The co-propagating reference pulse is not shown. 

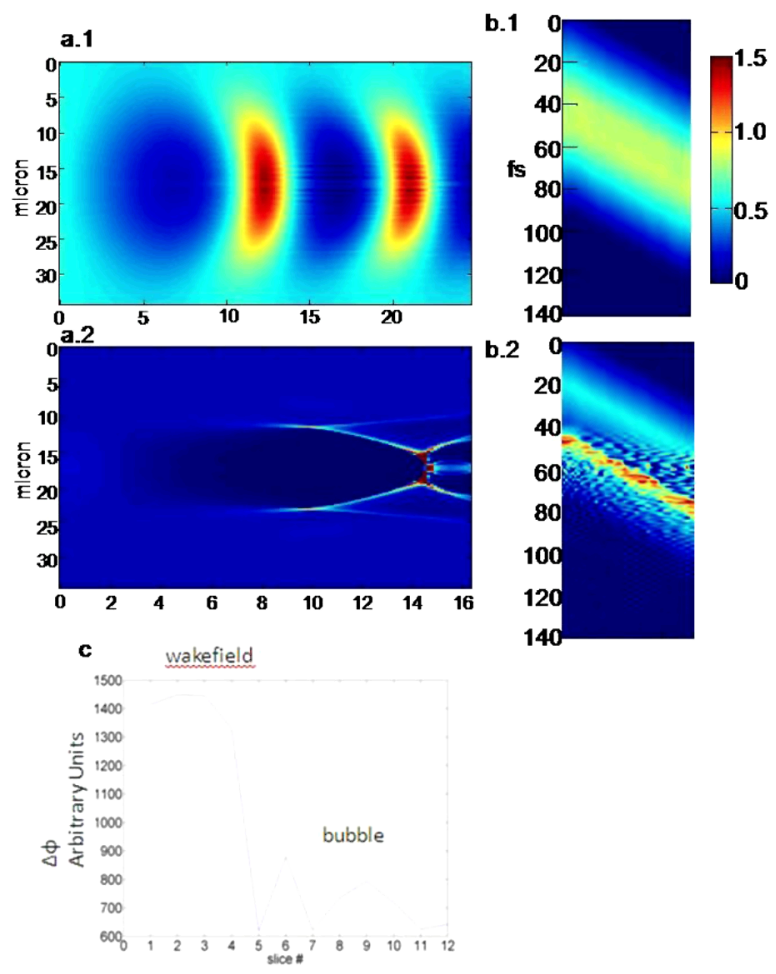

FIGURE 2. (a) Plasma density simulated by WAKE. a.1 is the wakefield stage. a.2 is bubble stage. (b) Probe pulse phase map. The $\mathrm{y}$ axis is the duration of the probe. The $\mathrm{x}$ axis is the transverse size of the probe. The phase map clearly shows the difference between the wakefield and bubble stage. (c) Each point on the line is the sum of a small region on figure 2.b. For different snapshots, same region is summed. When bubble forms, there is a big change in phase magnitude.

A simulation using the PIC code $\mathrm{WAKE}^{5}$ and the commercial finite element code COMSOL was carried out to generate a detailed, quantitative phase streak on a probe pulse for realistic experimental conditions. WAKE simulated the axi-symmetric wake structure generated by a pump pulse with Gaussian radial and temporal profile focused at the gas jet entrance. Simulation parameters were: $\mathrm{a}_{0}=2.15$; beam radius $=16$ micron; plasma density $\mathrm{n}_{\mathrm{e}}=1.5 \times 10^{19} / \mathrm{cm}^{-3}, \lambda=800 \mathrm{~nm}$. In total, snapshots of plasma density were generated at 12 different times of pump pulse propagation, encompassing various stages of bubble evolution. COMSOL then solved the time dependent 2-D Maxwell equations for the oblique propagation of a $400 \mathrm{~nm}$ probe pulse through the plasma. The probe pulse was simulated by introducing a time varying boundary current at the left edge of simulation region. The bubble propagated from the top to the bottom of the simulation region. The index of refraction of the simulation region was updated every femtosecond and on every grid point. After passing through the plasma, the probe pulse electric field intensity was recorded and the electric field phase extracted by an FFT method. Figure 2a shows two of the 12 WAKE simulation results. Fig. 2a.1 is the plasma structure immediately after the laser pulse entered the 
plasma, where it formed a sinusoidal wakefield. Fig. $2 \mathrm{a} .2$ is the structure after the pulse has propagated half-way through the plasma, self-focused and self-steepened, and formed a bubble. Fig. 2b shows COMSOL simulations of the corresponding sections of the phase streak imprinted on the probe pulse. In Figure 2.b.1, peaks and valleys of the wake are averaged out making it difficult to identify the period of the wakefield. In Figure 2.b.2, the head and the tail of the bubble is clearly shown in the phase streak. This distinctive difference between these two sections of the phase streak makes it effortless to spot the onset of the bubble formation. Fig. 2c, a lineout of $\Delta \phi$ near the axis of the phase streak, further illustrates the dramatic change in $\Delta \phi$ that accompanies bubble formation.

The drawback of FDSC by itself is that it records only a 1-D projection of a 2-D cross section of the spatial structure because the probe phase is integrated in the direction of the structure movement. Consequently the shape of the bubble at each $z$ is still largely unknown.

\section{FREQUENCY DOMAIN TOMOGRAPHY}

To overcome this drawback, we propose Frequency Domain Tomography (FDT). The basic concept of computer-aided tomography (CAT) is to record a series of projections of a $3 \mathrm{D}$ volume onto a $2 \mathrm{D}$ image surface, each at a different viewing angle. Each horizontal line within a film gives a 1-D projection of a 2-D axial cross-section of the 3D volume at that angle. Thus, a collection of horizontal lines, one from each film at the same height, contains information about only one axial cross-section. These projection data are then used to reconstruct cross-sectional images. Here we utilize the same principle to reconstruct a 'bubble' from phase streaks taken from probe pulses at different angles.

FDT and CAT differ in that the former records accumulated phase of a moving object, the latter of a stationary object. To make the connection between FDT and CAT, consider a reference line $\xi_{\perp}$ on the probe in the plane of Fig. $1 \mathrm{~b}$ perpendicular to the direction $\xi$ shown in Fig. $1 b$ that remains stationary in the reference frame of the probe. A bubble of radius $r_{b}$ crosses this line at right angles with velocity $\mathrm{v}=$ $2 \operatorname{csin}(\alpha / 2)$, i.e. with transit time $\tau_{\text {transit }}=r / c \sin (\alpha / 2)$. For example, the bubble in our simulation has radius $r_{b}=20 \mu \mathrm{m}$, so it crosses the reference line in $\tau_{\text {transit }}=94 \mathrm{fs}$ for $\alpha$ $=90^{\circ}$. Since the bubble's total propagation time across a $1 \mathrm{~mm}$ gas jet is $\sim 3.3 \mathrm{ps}$, it is reasonable to assume that the bubble's structure remains quasi-static during this transit time. With this mild assumption, the accumulated phase profile $\Delta \phi\left(\xi_{\perp}\right)$ along the reference line $\xi_{\perp}$ is identical to the phase profile that would be accumulated by the same probe propagating across an identical stationary bubble at angle $\pi / 2-\alpha / 2$ with respect to its front-back axis. The existence of this equivalent problem establishes the connection between FDT and CAT, and enables the use of filtered back projection and reconstruction techniques already developed for clinical CAT scans ${ }^{6}$ with little or no alteration at all.

To complete space-time reconstruction of the bubble, additional reference lines parallel to $\xi_{\perp}$ are constructed along the phase streak at spacings $\geq \tau_{\text {transit. }}$. Phase profiles along these lines represent 1-D projections of quasi-static bubble structure at different 
stages of evolution. We then acquire an equivalent family of phase profiles from streaks recorded at different intersection angles $\alpha$, and apply the clinical CAT algorithm. Figure 3 shows simulation results for tomographic reconstruction of one representative evolutionary stage of a bubble using different numbers of probe pulses in the same plane. Two or three probe beams are enough to detect the head and tail of the plasma bubble because of high electron density on those parts. For the whole bubble shape, 10 probe beams are necessary. Applying more probe beams from different planes, a 3-D reconstruction at each evolutionary stage is also possible as long as the probe beams do not perturb the plasma.
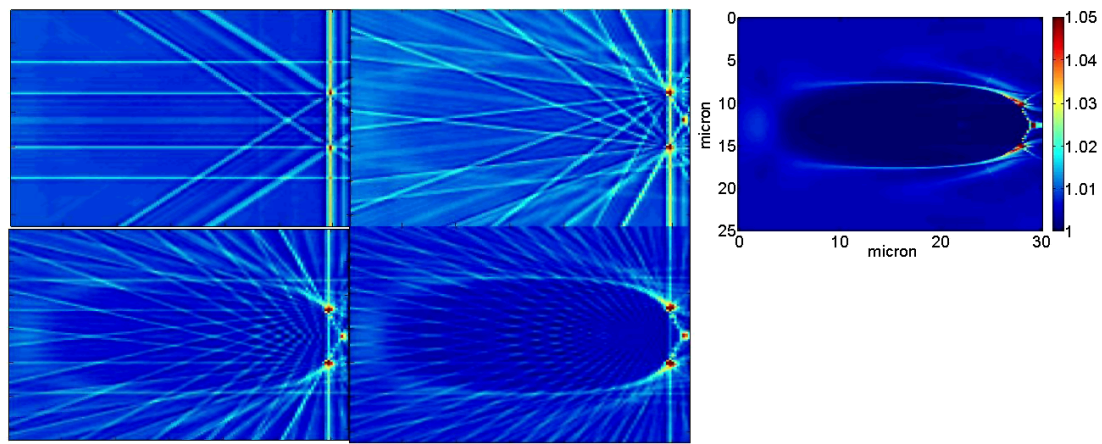

FIGURE 3. Using filtered back projection technique, different numbers of probe beams at various angles are tested to reconstruct bubble shape for a chosen bubble which is presented above. Top left: 3 beams. Top right: 5 beams. Bottom left: 9 beams. Bottom right: 18 beams.

\section{CONCLUSION}

The Frequency Domain Streak Camera provides a new way of recording time evolution and 1-D spatial projection of a plasma structure using the techniques of FDH. Frequency Domain Tomography, a further generalization of FDSC, will provide 2-D or even 3-D space-time reconstruction of evolving plasma structures.

\section{ACKNOWLEDGMENTS}

This work was supported by U. S. DOE grants DEFG03-96-ER-40954, DE-FG0204ER54763, DE-FG02-04ER41321, and DE-FG02-07ER54945.

\section{REFERENCES}

1. N. H. Matlis et al., Nature Physics 2, 749 (2006).

2. S. P. Le Blanc et al., Opt. Lett. 25, 764 (2000).

3. A. Pukhov, Plasma Phys. Control. Fusion 46, B179 (2004).

4. J. Faure, Y. Glinec, J. J. Santos et al., Phys. Rev. Lett. 95, 205003 (2005).

5. P. Mora \& T. Antonsen, Phys. Plasmas 4, 217 (1997).

6. R. A. Brooks \& G. DiChiro, Phys. Med. Biol. 21, 689 (1976). 